\title{
Thymocyte selection: CD8 helps TCR catch slippery self pMHC
}

\author{
Omer Dushek $^{1}$ and Michael L. Dustin ${ }^{2}$
}

1Sir William Dunn School of Pathology, University of Oxford, South Parks Road, OX1 3RE (ORCID: https://orcid.org/0000-0001-5847-5226)

2The Kennedy Institute of Rheumatology, Roosevelt Dr, Oxford, OX3 7FY (ORCID: https://orcid.org/0000-0003-4983-6389)

\section{A new model proposes that an applied force allows the CD8 coreceptor and the kinase Lck to selectively stabilize TCR interactions with negatively, but not positively selecting ligands, during the negative selection of self- reactive thymocytes.}

Development of conventional T cells requires immature T cells (thymocytes) to rearrange the $\beta$ and then $\alpha$ subunits of the T cell receptor (TCR) and test the expressed TCR for their ability to interact with peptide-major histocompatibility complexes (pMHC) expressed on the surface of thymic epithelial cells and dendritic cells. Positive selection takes place when thymocytes, which express both CD4 and CD8, detect "weak" interaction of their TCR with pMHC with help of either co-receptor. If the TCR signal exceeds a threshold that indicates a dangerous level of self-reactivity the thymocyte undergoes apoptosis, removing the threat. Up to now, there was no clear separation in any parameter of TCRpMHC interaction that adequately separated ligands between those inducing negative and positive selection. Discrimination was explained through purely biochemical kinetic proofreading models, that would amplify small kinetic differences ${ }^{2}$. In this issue of Nature Immunology, Hong et al. show that under mechanical force, the CD8 co-receptor and the kinase Lck generate a clear physical separation between negative and positive selecting pMHC through formation of a dynamic "catch" bond only by the negatively selecting pMHC ${ }^{1}$.

Catch bonds are a class of molecular interactions involved in cell adhesion and motility and predicted to display increased lifetimes when subjected to force ${ }^{3}$. This contrasts with most molecular interactions, which undergo accelerated dissociation when subjected to forces, and are referred to as "slip" bonds. For example, atomic force microscopy studies on selectins, which mediate rolling adhesion in blood vessels, demonstrate an intrinsic capacity to increase their lifetime in response to $\sim 10 \mathrm{pN}$ forces ${ }^{4}$. It is important to point out that there are no perfect catch bonds; as force increases, all interactions eventually slip, and display faster dissociation above a certain force. The typical depiction of a catch bond is that the lifetime of the bond increase with force to a peak near 10-20 pN force, then drops back toward 0 as force is further increased (Fig. 1a). A welldefined system that displays such behavior is the interaction of von Willebrand factor with platelet glycoprotein Ib $\alpha$ during blood clotting, which displays an effective catch bond by a force-dependent extension ${ }^{5}$. This type of catch bond was termed a "flex bond", to highlight the force-induced allosteric extension. 
Agonistic TCR-pMHC interactions undergo a $10 \mathrm{~nm}$ extension under force, so they also fits the criteria for flex bonds ${ }^{6}$. The innovation in the work of Hong et al. was to show that the CD8 co-receptor and the TCR independently formed slips bonds with pMHC, but when acting in parallel, a catch bond appeared only for negatively selecting ligand that required active input from the thymocyte ${ }^{1}$.

Hong et al. used a biomembrane force probe, a micromanipulation system that uses a red blood cell as a pN spring to present a bead coated with pMHC to live CD $4{ }^{+} \mathrm{CD}^{+}$double positive thymocytes ${ }^{1}$. By contacting and retracting the thymocyte under conditions where less than $10 \%$ of trials generate an interaction, a defined force can be generated on the TCR-pMHC interaction and the lifetime of the single bond can be measured. By repeated cycles, many such lifetimes can be determined and averaged for different values of the applied force (from 0 to $\sim 30 \mathrm{pN}$ ). Unexpectedly, positively selecting ligands showed a slip bond behavior, whereas negatively selecting ligands showed a catch bond behavior (Fig. 1a,b). The catch bond behavior was dependent on coreceptor binding and on the catalytic activity of Lck. In fact, CD8-CD4 chimeras with greater Lck binding converted a positively selecting pMHC into a negatively selecting pMHC, including catch bond formation (Fig. 1a). The molecular model that emerges, termed 'dynamic catch bond model' by the authors, invokes CD8associated Lck phosphorylating and then binding the TCR cytoplasmic domain to form the catch bond by increasing rebinding within the TCR-pMHC-CD8 trimer (Fig. 1b) ${ }^{1}$. An applied force of $\sim 10 \mathrm{pN}$ increases the lifetime of negatively selecting ligands, but decreases the lifetime of positively selecting ligands leading to their lifetimes being well separated (Fig. 1a).

Although force creates a greater separation between positive and negative selecting ligands, there is still overlap in the lifetime distributions. Hong et al. perform a statistical calculation to show that $>32$ sequential binding events with force enable thymocytes to accumulate sufficient information to determine whether they should survive or die ${ }^{1}$. However, selection using the lifetime distributions without force or without the coreceptor produces poor discrimination, because the measured distributions overlap extensively. This statistical calculation is consistent with the induced rebinding model of antigen discrimination, whereby TCR-pMHC binding for sufficiently long periods of time induces processes such as coreceptor binding and/or TCR clustering, that would promote the immediate rebinding of $\mathrm{pMHC}^{7}$.

The dynamic catch bond model invokes force as the mechanism that increases the rebinding of each molecule within a trimer that consists of individual slip bonds maintained in part by the action of $\mathrm{Lck}^{1}$. Clustering interactions are also known to result in a longer effective bond lifetime ${ }^{8}$ and therefore clustering may generate an apparent catch bond from slip bonds. Examination of the survival probability curves presented by Hong et al. ${ }^{1}$ suggest another view in which force might select Lck-stabilized long-lived bonds by rapidly eliminating short-lived bonds in the force clamp assay, which we could call a force-selection model. Since in this model all interactions would be slip bonds, a possible way to distinguish these alternative views would be to directly link CD8 to the TCR 
(bypassing Lck) in order to determine whether this complex is intrinsically a slip or catch bond.

The role of Lck suggests that rapid, active processes participate in dynamic catch bond formation. The dynamics of immunological synapse, including structures formed by thymocytes, provide an environment rich in forces ${ }^{9}$. The formation of F-actin-dependent microclusters, rather than initial single molecule interactions that formed similarly for both catch and slip bonds, was revealed as the step that distinguished bimolecular TCR-pMHC catch bonds from slip bonds in the synapse ${ }^{10}$. In fact, a large class of pMHC that bound with TCR in solution assays formed slip bonds and failed to stimulate in a mature immunological synapse setting. Thus, Hong et al. ${ }^{1}$ have expanded our appreciation of how mechanical force enters into developmental decision-making by incorporating active processes such as signaling in split second time frames. Its clear that force will need to be considered as a parameter in all stages of adaptive responses, including building the repertoire.

1. Hong, J. et al. Outside-in/inside-out signaling loop of TCR mechanotransduction induced by negative selecting ligands in the thymus. Nat. Immunol. (2018).

2. Stepanek, O. et al. Coreceptor scanning by the T cell receptor provides a mechanism for T cell tolerance. Cell 159, 333-345 (2014).

3. Dembo, M., Torney, D.C., Saxman, K. \& Hammer, D. The reaction-limited kinetics of membrane-to-surface adhesion and detachment. Proceedings of the Royal Society of London. Series B, Containing papers of a Biological character. Royal Society 234, 55-83 (1988).

4. Marshall, B.T. et al. Direct observation of catch bonds involving celladhesion molecules. Nature 423, 190-193 (2003).

5. Kim, J., Zhang, C.Z., Zhang, X. \& Springer, T.A. A mechanically stabilized receptor-ligand flex-bond important in the vasculature. Nature 466, 992995 (2010).

6. Das, D.K. et al. Force-dependent transition in the T-cell receptor betasubunit allosterically regulates peptide discrimination and $\mathrm{pMHC}$ bond lifetime. Proc Natl Acad Sci U S A 112, 1517-1522 (2015).

7. Dushek, O. \& van der Merwe, P.A. An induced rebinding model of antigen discrimination. Trends Immunol 35, 153-158 (2014).

8. Chen, S. \& Springer, T.A. An automatic braking system that stabilizes leukocyte rolling by an increase in selectin bond number with shear. J Cell Biol 144, 185-200 (1999). 
9. Hailman, E., Burack, W.R., Shaw, A.S., Dustin, M.L. \& Allen, P.M. Immature CD4(+)CD8(+) thymocytes form a multifocal immunological synapse with sustained tyrosine phosphorylation. Immunity 16, 839-848. (2002).

10. Sibener, L.V. et al. Isolation of a Structural Mechanism for Uncoupling T Cell Receptor Signaling from Peptide-MHC Binding. Cell 174, 672-687 e627 (2018).

A

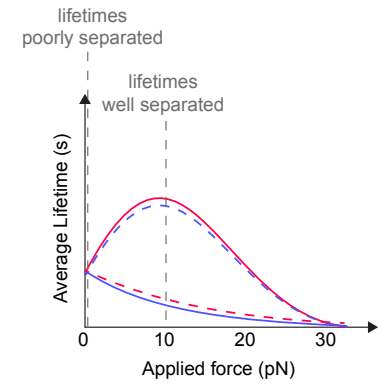

positively selecting ligand (slip bond)

_ negatively selecting ligand (catch bond)

. - . negatively selecting ligand without coreceptor (slip bond)

. - . - positively selecting ligand with enhanced coreceptor (catch bond)

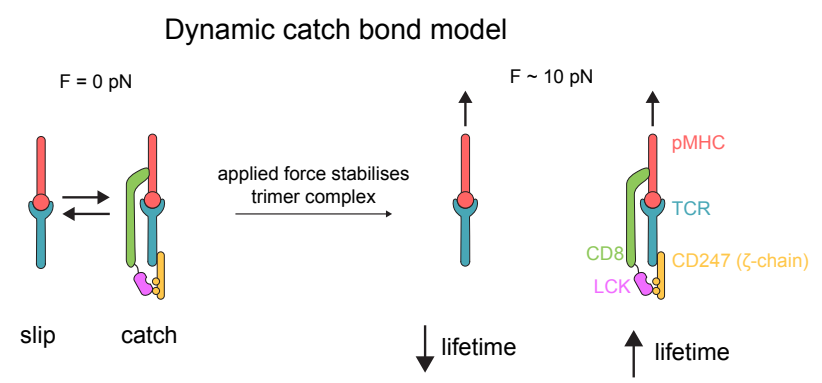

Figure 1. Molecular forces separate the lifetime of positively and negatively selecting ligands. a) The lifetime of positively and negatively selecting ligands are similar without force $(\mathrm{F}=0)$. Hong et $\mathrm{al}^{1}$ now show that these lifetimes become well separated under an applied force $(\mathrm{F} \sim 10 \mathrm{pN})$. Positively selecting ligands formed a slip bond so that an applied force decreased the lifetime (solid blue) whereas negatively selecting ligands formed a catch bond so that an applied force increased the lifetime (solid red). The catch bond is abolished if a mutation is introduced to the MHC to prevent CD8 binding or if the catalytic activity of Lck is inhibited (dashed red). The slip bond can be converted to a catch bond using a co-receptor chimera with increased Lck association (dashed blue). b) The dynamic catch bond model proposes that the trimer complex is a catch bond whereby an applied force increases rebinding between TCR, CD8 and pMHC leading to a longer-lived complex, even though the TCR-pMHC and the CD8pMHC interactions are slip bonds. By inducing more trimers, negatively selecting ligands produce more stabilised complexes under force. 